\title{
CHROMATOGRAPHIA: Its Position in the Fast Changing World of Analytical Sciences
}

\author{
Henk Lingeman ${ }^{1}$
}

(C) Springer-Verlag Berlin Heidelberg 2016

CHROMATOGRAPHIA is a scientific journal focused on the communication of the separation sciences in all their aspects. However, in the previous century the separation sciences were mainly limited to chromatographic and electrophoretic approaches using mono-dimensional systems. In the new millennium, hyphenated and/or integrated systems have become the norm in analytical-based approaches (see Fig. 1).

This means that in addition to mass spectrometric, spectroscopic, and electrochemical approaches, biological procedures (e.g., enzymatic, immunological, receptor-based assays) are also part of the whole analytical process. In the future, CHROMATOGRAPHIA will focus on new developments in sorbent materials and techniques to improve the selectivity, efficiency and speed of separation devices, on hyphenated systems that combine in an integrated way several unit operations (e.g., sample clean-up, separation, assay, detection) to construct (fully) automated systems that fulfill the requirements of the users (speed, reliability, miniaturization) and on the scaling down of existing systems to enable the analysis of smaller sample sizes and volumes and to allow the simultaneous screening of multiple compounds (i.e., -omics approaches). As a consequence a primary focus will be on the integration of several unit operations in a single analytical instrument. In these areas preliminary data can be published as long as there is sufficient novelty in the various unit operations or their combination and the results will be beneficial for the readers of

Henk Lingeman

h.lingeman@vu.nl

1 VU University Amsterdam, BioAnalytical Chemistry, De Boelelaan 1108, 1081 HZ Amsterdam, The Netherlands
CHROMATOGRAPHIA and could stimulate them to bring their own research to a higher level.

Examples of the type of papers CHROMATOGRAPHIA would like to focus on, are the recent review papers: Highthroughput analysis and automation for glycomic studies [1]; Microfluidic paper-based analytical devices ( $\mu$ PADs) and micro total analysis systems ( $\mu \mathrm{TAS}$ ): development, applications and future trends [2] and the original papers: Layer-by layer assemblies based on a cationic $\beta$-cyclodextrin polymer: chiral stationary phases for open-tubular electrochromatography [3], The development of the in situ modification of 1st generation analytical scale silica monoliths [4].

To assist this process CHROMATOGRAPHIA will be offering flexibility in manuscript handling and an enhanced way of communication with the authors and the readers by offering accelerated publication for high quality papers. Authors will have the opportunity to submit an original manuscript that is within the primary scope of the journal and to ask for accelerated publication. One of the Editors will decide within three working days if the paper has sufficient quality and will send it out for reviewing, and after five further working days a decision on the manuscript will be made by the Editor and communicated to the authors and if accepted publication will follow as soon as possible. The length of the manuscripts can be between 2 and 10 pages.

To support this approach the journal will also encourage short review-type papers dealing with a particular topic which is of importance for the readers of CHROMATOGRAPHIA (e.g., integration, hyphenation, miniaturization, stationary phases, theoretical discussions, detection). Authors are invited to submit relatively short papers dealing with critical comparisons between various approaches, unit operation, systems, etc.

Since separations are only valuable when they are combined with selective and/or sensitive detection, discussions 
Fig. 1 Example of a hyphenated/integrated system in which a chromatographic separation procedure is integrated with an enzymatic bioassay and mass spectrometry as the detection mode

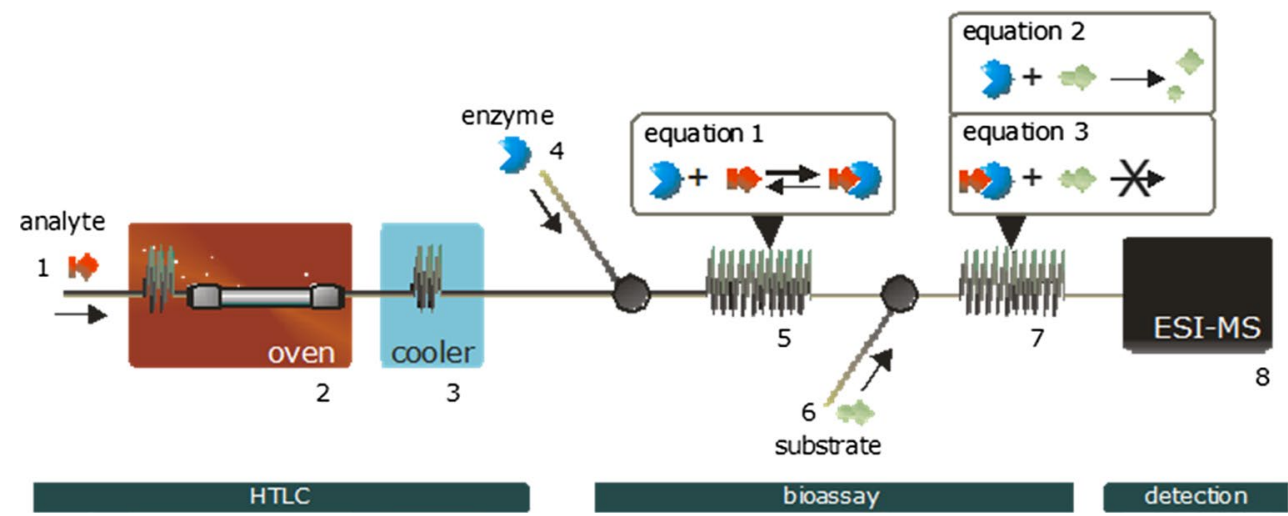

on the theoretical and practical aspects of mass spectrometry, amperometry, fluorescence, enzymology or immunology are certainly within the scope of CHROMATOGRAPHIA. Advances in the integrated combinations of chromatography or electrophoresis with advanced mass spectrometric devices for the identification and quantitation of multiple compounds in complex samples, interfacing designs between various separation and detection systems, the integration with nano technology (chip-based designs) with separation systems and the miniaturization of sample preparation and clean-up approaches will be becoming more and more important the future, and will, therefore, form the backbone of the journal.

As Editors we hope that by focusing on modern technology on one hand, and on the practical and theoretical fundamentals on the other hand and less on the application side of separation sciences CHROMATOGRAPHIA will become a really valuable tool for our readers that are dealing with the understanding and development of separationbased approaches.

On behalf of the Scientific Editors of CHROMATOGRAPHIA.

Henk Lingeman

\section{References}

1. Shubhakar A, Reiding KR, Gardner RA et al (2015) Chromatographia 78:321-333

2. Lisowski P, Zarzycki PK (2013) Chromatographia 76:1201-1214

3. Pédehontaa-Hiaa G, Guerrouache M, Carbonnier B et al (2015) Chromatographia 78:533-541

4. Soliven A, Dennis GR, Hilder EF et al (2014) Chromatographia 77:663-671 